\title{
First Identification of a Triple Corneal Dystrophy Association: Keratoconus, Epithelial Basement Membrane Corneal Dystrophy and Fuchs' Endothelial Corneal Dystrophy
}

\author{
Cosimo Mazzotta ${ }^{\mathrm{a}} \quad$ Claudio Traversi $^{\mathrm{a}} \quad$ Frederik Raiskup $^{\mathrm{c}}$ \\ Caterina Lo Rizzo ${ }^{b} \quad$ Alessandra Renieri ${ }^{b}$ \\ ${ }^{a}$ Department of Ophthalmology, and ${ }^{b}$ Medical Genetics, University of Siena, Siena, Italy; \\ ${ }^{\mathrm{C}}$ Department of Ophthalmology, Technical University of Dresden, Dresden, Germany
}

\section{Key Words}

Keratoconus · Fuchs' endothelial corneal dystrophy · Epithelial basement membrane dystrophy $\cdot$ Cogan dystrophy $\cdot$ Confocal microscopy $\cdot$ ZEB1

\begin{abstract}
Purpose: To report the observation of a triple corneal dystrophy association consisting of keratoconus $(\mathrm{KC})$, epithelial basement membrane corneal dystrophy (EBMCD) and Fuchs' endothelial corneal dystrophy (FECD). Methods: A 55-year-old male patient was referred to our cornea service for blurred vision and recurrent foreign body sensation. He reported bilateral recurrent corneal erosions with diurnal visual fluctuations. He underwent corneal biomicroscopy, Scheimpflug tomography, in vivo HRT confocal laser scanning microscopy and genetic testing for TGFBI and ZEB1 mutations using direct DNA sequencing. Results: Biomicroscopic examination revealed the presence of subepithelial central and paracentral corneal opacities. The endothelium showed a bilateral flecked appearance, and the posterior corneal curvature suggested a possible concomitant ectatic disorder. Corneal tomography confirmed the presence of a stage II KC in both eyes. In vivo confocal laser scanning microscopy revealed a concomitant bilateral EBMCD with hyperreflective deposits in basal epithelial cells, subbasal Bowman's layer microfolds and ridges with truncated subbasal nerves as pseudodendritic elements. Stromal analysis revealed honeycomb edematous areas,
\end{abstract}

Cosimo Mazzotta, MD, PhD

Policlinico Le Scotte, U.O.C. Oculistica

Viale Mario Bracci

IT-53100 Siena (Italy)

E-Mail cgmazzotta@libero.it 
Mazzotta et al.: First Identification of a Triple Corneal Dystrophy Association: Keratoconus, Epithelial Basement Membrane Corneal Dystrophy and Fuchs' Endothelial Corneal Dystrophy

and the endothelium showed a strawberry surface configuration typical of FECD. The genetic analysis resulted negative for TGFBI mutations and positive for a heterozygous mutation in exon 7 of the gene ZEB1. Conclusion: This is the first case reported in the literature in which $K C, E B M C D$ and FECD are present in the same patient and associated with ZEB1 gene mutation. The triple association was previously established by means of morphological analysis of the cornea using corneal Scheimpflug tomography and in vivo HRT II confocal laser scanning microscopy.

(C) 2014 S. Karger AG, Basel

\section{Introduction}

Corneal dystrophies refer to a group of bilateral, genetically determined, corneal diseases characterized by a progressive loss of corneal transparency that results from the accumulation of deposits within the different corneal layers. Age at onset, as in most inherited eye disorders, is variable. Corneal dystrophies are classified according to their slit lamp appearance, the morphology of the deposits, the depth of the corneal involvement and their histopathological features [1].

Most of the corneal dystrophies are of Mendelian inheritance with variable expressivity and reduced penetrance [2]. In some cases, such as keratoconus (KC; MIM 148300), the inheritance pattern appears to be more complex because both genetic and environmental factors play a role in the etiology of this disease [3-6]. However, the VSX1 gene remains the best-characterized KC gene. Mutations in VSX1 were also described in the association between posterior polymorphous corneal dystrophy (PPCD; MIM 122000) and KC [7, 8]. Recently, mutations in the ZEB1 gene have been reported as a cause of 30\% of PPCD cases [9].

Furthermore, ZEB1 is involved in few cases of Fuchs' endothelial corneal dystrophy (FECD; MIM 136800) and also in rare cases with an association of FECD and KC [8-10]. Epithelial basement membrane corneal dystrophy (EBMCD; MIM 121820), also known as Cogan microcystic epithelial dystrophy or map-dot-fingerprint dystrophy, represents a common bilateral epithelial dystrophy [11]. Several families with autosomal dominant inheritance have been described and most EBMCD patients have a mutation in the TGFBI gene. Almost all types of EBMCD have been demonstrated to be due to different mutations in TGFBI with specific genotype-phenotype correlations [11,12].

Clinical and in vivo microscopic [13] identification of these coexisting conditions leads to a reasonable genetic link between distinct phenotypic entities with different prognoses and therapeutic requirements. We report a triple corneal dystrophy association consisting of KC, EBMCD and FECD described by biomicroscopy, Scheimpflug corneal tomography and in vivo HRT confocal laser scanning microscopy.

\section{Case Report}

A 55-year-old male patient was referred to our cornea service for blurred vision and recurrent foreign body sensation. The patient reported bilateral recurrent corneal erosions with bilateral visual fluctuations. Corrected distance visual acuity was 20/40 ( -2 cylinder axis $\left.70^{\circ}\right)$ and 20/60 $\left(+0.50\right.$ sphere $=-3$ cylinder axis $70^{\circ}$ int.) in the right and left eye, respectively. Biomicroscopic examination revealed the presence of subepithelial central and paracentral corneal opacities. The endothelium was altered with a bilateral flecked 
Mazzotta et al.: First Identification of a Triple Corneal Dystrophy Association: Keratoconus, Epithelial Basement Membrane Corneal Dystrophy and Fuchs' Endothelial Corneal Dystrophy

appearance, and the posterior corneal curvature suggested a possible ectatic disorder (fig. 1).

Initial lens nucleus opacities were present in both eyes. Endothelial cell count was 1,110 and 980 cells $/ \mathrm{mm}^{2}$ in the right and left eye, respectively. Corneal tomography confirmed the presence of a stage II KC in both eyes (fig. 2).

In vivo confocal laser scanning microscopy of the corneas unexpectedly revealed the presence of a concomitant EBMCD with hyperreflective deposits in basal epithelial cells, subbasal Bowman's layer microfolds and ridges with truncated subbasal nerves. Typical variously shaped linear, curvilinear or ring-shaped geographic hyperreflective materials were observed mainly in the basal epithelial cell layer (maps). Anterior keratocytes showed signs of activation with highly reflective nuclei, and uneven increased deposition of abnormal extracellular matrix in the anterior stroma was detected. The subbasal nerve plexus showed various pathologic changes, such as strange short nerve fiber bundles similar to dendritic Langerhans cells with decreased numbers of long nerve fibers. Fingerprint multiple linear and curvilinear hyporeflective lines were also observed. Additionally, highly reflective Langerhans cell-like or dot images were recorded. The stroma revealed honeycomb edematous areas, and the endothelium showed a strawberry surface configuration typical of FECD (fig. 3, fig. 4).

Genomic DNA was extracted from peripheral blood leukocytes using a QIAamp DNA Blood Maxi Kit, according to the manufacturer's protocol (Quiagen, Hilden, Germany). Whereas TGFBI is a commonly mutated gene in EBMCD, we performed, using direct sequencing, the research of the three best-known mutations (Arg555Trp, Arg124Leu and Ala546Asp) described in TGFBI and associated with corneal dystrophies. The analysis resulted negative. The genetic analysis resulted positive in a heterozygous pathogenic mutation in exon 7 (c.1920G>T; p.Gln640His) of the gene ZEB1.

\section{Discussion}

The TGFBI gene is mainly expressed in the corneal epithelium, and its product, keratoepithelin, regulates cell adhesion. The discovery that mutations in the TGFBI gene are the cause of a group of corneal dystrophies (granular type I, type II, lattice and Reis-Bücklers) has changed our knowledge of these conditions. Since this finding, the genetic basis of many corneal dystrophies has been described $[2,12]$.

A recent paper [8] found that missense substitutions in the ZEB1 protein are associated with FECD and KC, whereas protein-truncating ZEB1 mutations result in PPCD. We found instead a case (the first one reported in the literature) in which the three pathologies are present in the same patient and associated with the mutation of one known gene (ZEB1). In that study, RT-qPCR was also performed on cultured corneal keratocytes harboring the missense ZEB1 mutation (p.Gln640His), demonstrating that COL4A1 and COL4A2 were markedly downregulated, and COL4A3, COL4A4, and COL8A2 were moderately downregulated [7-9]. We could not assess the aforementioned downregulation due to the unavailability of the proper analysis in our laboratory.

It is known that these corneal dystrophies are characterized by genetic heterogeneity: a specific gene causes several conditions and, on the other hand, specific gene mutations determine specific phenotypes. For this reason, according to the IC3D classification [1], the diagnosis of corneal dystrophy today requires genetic evidence [2]. 
Mazzotta et al.: First Identification of a Triple Corneal Dystrophy Association: Keratoconus, Epithelial Basement Membrane Corneal Dystrophy and Fuchs' Endothelial Corneal Dystrophy

However, while waiting for genetic testing, there are clinical and instrumental observations using biomicroscopy, corneal tomography and in vivo confocal analysis that may help the diagnosis with a certain reliability [1].

In this clinicopathological report the triple association consisting of KC, EBMCD and FECD was clearly established by means of morphological analysis of the cornea using corneal tomography and in vivo HRT confocal laser scanning microscopy. These coexisting conditions have different prognoses according to their natural history, progression rate and patient's age. KC in a 55-year-old male patient rarely progresses and does not represent a clinical problem according to the patient's functional data. EBMCD with recurrent epithelial erosions seems to be responsible for the patient's main clinical symptoms and corneal opacities; however, it can be managed by medical therapy (eye lubricants, therapeutic soft contact lens bandage, autoserum, amniotic membrane) or at least minimal excimer laser phototherapeutic keratectomy.

The most relevant problem is related to FECD, which must be carefully monitored according to the patient's age and cataract diagnosis. In this case the corneal thinning caused by KC can mask the severity of endothelial disease. The association between KC and FECD may combine to normalize the corneal pachymetry readings that were 509 and $595 \mu \mathrm{m}$ on average for the right and left eye, respectively; disease severity may be underestimated, which may lead to unexpected postoperative visual outcomes. Moreover the severity of endothelial dystrophy may require a triple procedure (cataract extraction, IOL implant plus Descemet stripping automated keratoplasty).

Confocal microscopy in this case supported the diagnosis of EBMCD in both corneas. Although endothelial dystrophies, EBMCD and KC are clinically and pathologically distinct, this association suggests a common genetic basis. The absence of mutations in TGFBI, actually known as the main genetic cause of corneal dystrophies, could support the hypothesis of a new interfering gene. The genetic analysis indeed resulted positive in a heterozygous pathogenic mutation in exon 7 (c.1920G>T; p.Gln640His) of the gene ZEB1. ZEB1 is involved in FECD and also in rare cases with an association of FECD and KC [8-10]. In particular, ZEB1 missense substitutions are associated with FECD, whereas proteintruncating mutations result in PPCD [10]. The most recent literature pinpoints the function of ZEB1 in the dysregulation of collagen type IV alpha-3 production and suggests its role in the structure and function of cells lining the anterior chamber of the eye [10]. Thus, we can explain the link between ZEB1 mutation and the etiology of PPCD, FECD and KC.

Using genome-wide studies, the collection of patients with this specific association could allow discovering the currently unknown disease-causing genes. Anyway, complex pathological associations that may affect the cornea must be carefully distinguished according to different prognoses and therapeutic approaches. To our knowledge, this is the first report in the literature with this triple association.

\section{Disclosure Statement}

The authors declare no financial interest and have nothing to disclose. 
Mazzotta et al.: First Identification of a Triple Corneal Dystrophy Association: Keratoconus, Epithelial Basement Membrane Corneal Dystrophy and Fuchs' Endothelial Corneal Dystrophy

\section{References}

1 Weiss JS, Møller HU, Lisch W, Kinoshita S, Aldave AJ, Belin MW, Kivelä T, Busin M, Munier FL, Seitz B, Sutphin J, Bredrup C, Mannis MJ, Rapuano CJ, Van Rij G, Kim EK, Klintworth GK: The IC3D classification of the corneal dystrophies. Cornea 2008;27(suppl 2):S1-S83.

$\checkmark 2$ Vincent AL: Corneal dystrophies and genetics in the International Committee for Classification of Corneal Dystrophies era: a review. Clin Experiment Ophthalmol 2014;42:4-12.

-3 Poulaki V, Colby K: Genetics of anterior and stromal corneal dystrophies. Semin Ophthalmol 2008;23:9-17.

-4 Burdon KP, Vincent AL: Insights into keratoconus from a genetic perspective. Clin Exp Optom 2013;96:146154.

5 Salouti R, Nowroozzadeh MH, Zamani M, Ghoreyshi M: Combined anterior keratoconus and Fuchs' endothelial dystrophy: a report of two cases. Clin Exp Optom 2010;93:268-270.

-6 Bisceglia L, Ciaschetti M, De Bonis P, Campo PA, Pizzicoli C, Scala C, Grifa M, Ciavarella P, Delle Noci N, Vaira F, Macaluso C, Zelante L: VSX1 mutational analysis in a series of Italian patients affected by keratoconus: detection of a novel mutation. Invest Ophthalmol Vis Sci 2005;46:39-45.

7 Héon E, Greenberg A, Kopp KK, Rootman D, Vincent AL, Billingsley G, Priston M, Dorval KM, Chow RL, McInnes RR, Heathcote G, Westall C, Sutphin JE, Semina E, Bremner R, Stone EM: VSX1: a gene for posterior polymorphous dystrophy and keratoconus. Hum Mol Genet 2002;11:1029-1036.

-8 Lechner J, Dash DP, Muszynska D, Hosseini M, Segev F, George S, Frazer DG, Moore JE, Kaye SB, Young T, Simpson DA, Churchill AJ, Héon E, Willoughby CE: Mutational spectrum of the ZEB1 gene in corneal dystrophies supports a genotype-phenotype correlation. Invest Ophthalmol Vis Sci 2013;54:3215-3223.

$\$ 9$ Liskova P, Tuft SJ, Gwilliam R, et al: Novel mutations in the ZEB1 gene identified in Czech and British patients with posterior polymorphous corneal dystrophy. Hum Mutat 2007;28:638.

10 Vincent AL, Niederer RL, Richards A, Karolyi B, Patel DV, McGhee CN: Phenotypic characterisation and ZEB1 mutational analysis in posterior polymorphous corneal dystrophy in a New Zealand population. Mol Vis 2009;15:2544-2553.

11 Boutboul S, Black GC, Moore JE, Sinton J, Menasche M, Munier FL, Laroche L, Abitbol M, Schorderet DF: A subset of patients with epithelial basement membrane corneal dystrophy have mutations in TGFBI/BIGH3. Hum Mutat 2006;27:553-557.

12 Yang J, Han X, Huang D, Yu L, Zhu Y, Tong Y, Zhu B, Li C, Weng M, Ma X: Analysis of TGFBI gene mutations in Chinese patients with corneal dystrophies and review of the literature. Mol Vis 2010;16:1186-1193.

13 Shukla AN, Cruzat A, Hamrah P: Confocal microscopy of corneal dystrophies. Semin Ophthalmol 2012;27:107-116.

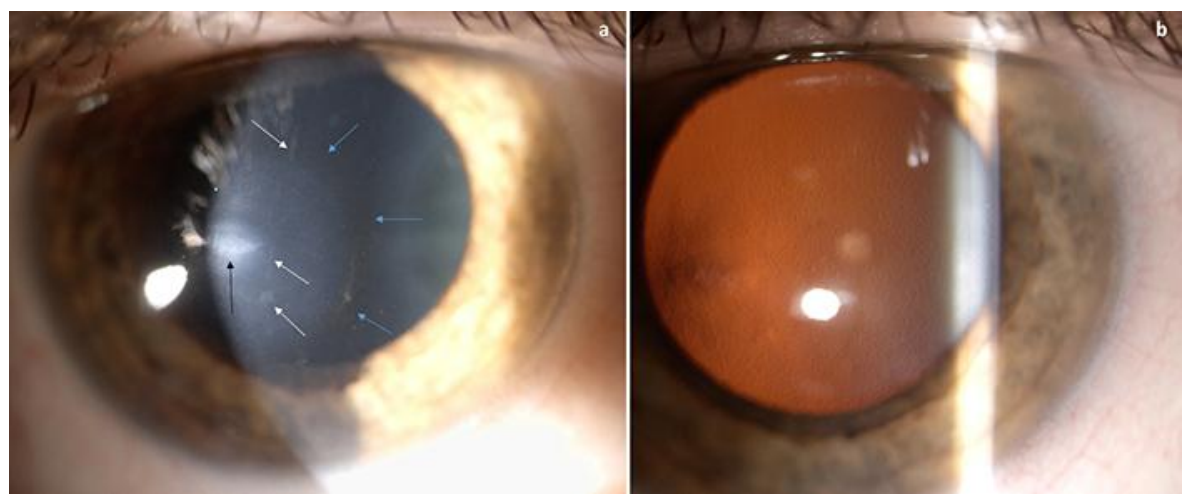

Fig. 1. a Slit lamp microscopy shows an oval scar (black arrow), multiple linear opacities (white arrows) and the Fleischer ring (light blue arrows). b Retroillumination technique clearly shows diffuse guttae and, on the left, the apex of KC. 


DOI: $10.1159 / 000367937$

(c) 2014 S. Karger AG, Basel

Mazzotta et al.: First Identification of a Triple Corneal Dystrophy Association: Keratoconus, Epithelial Basement Membrane Corneal Dystrophy and Fuchs' Endothelial Corneal Dystrophy

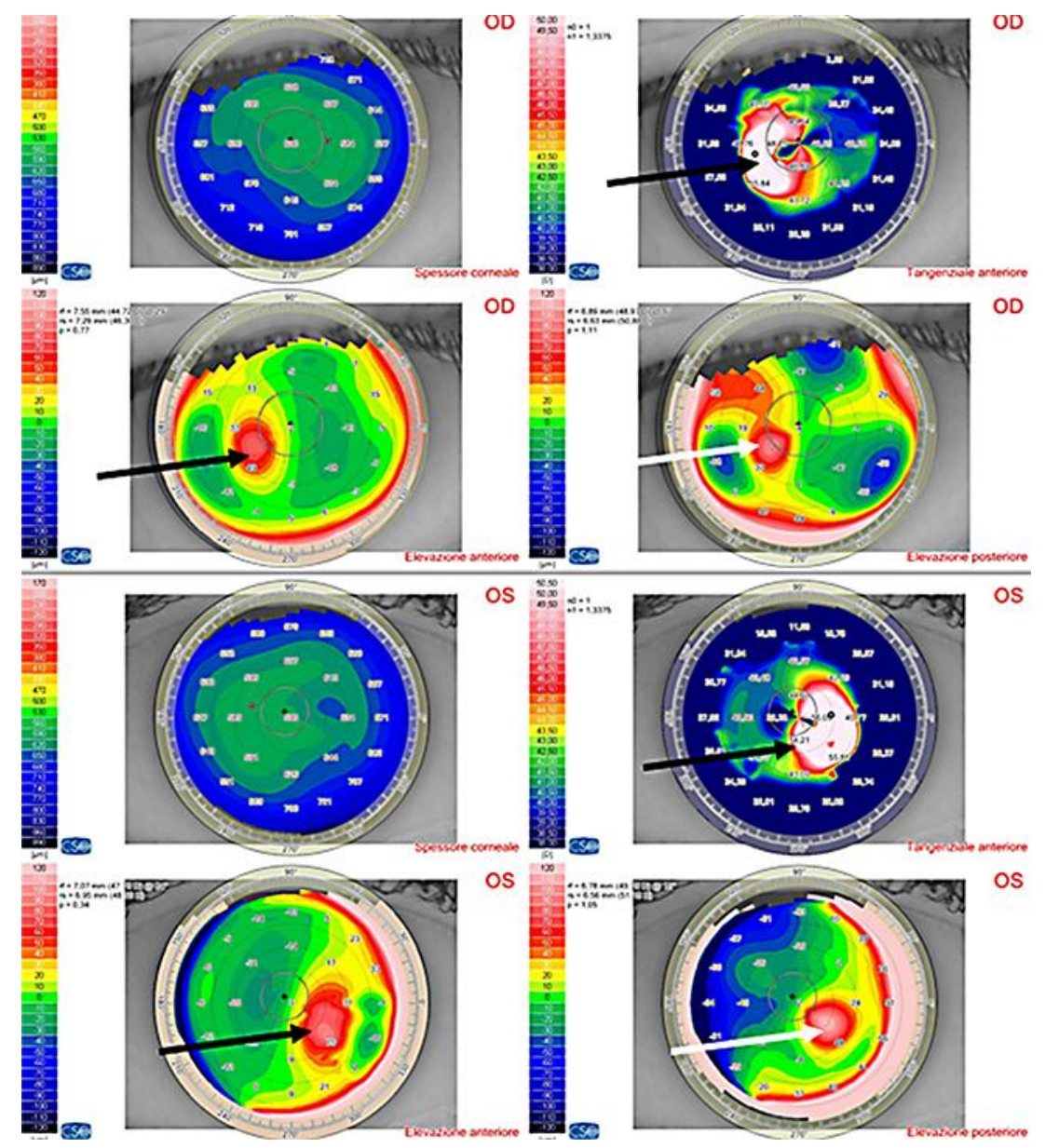

Fig. 2. Scheimpflug tomography shows KC in both eyes. Anterior tangential algorithm and elevation maps (black arrows); posterior elevation maps (white arrows). 


\section{Case Rep Ophthalmol 2014;5:281-288}

DOI: $10.1159 / 000367937$

lazzotta et al: First Identification of a Triple Corneal Dystrophy Associatior Keratoconus, Epithelial Basement Membrane Corneal Dystrophy and Fuchs' Endothelial Corneal Dystrophy

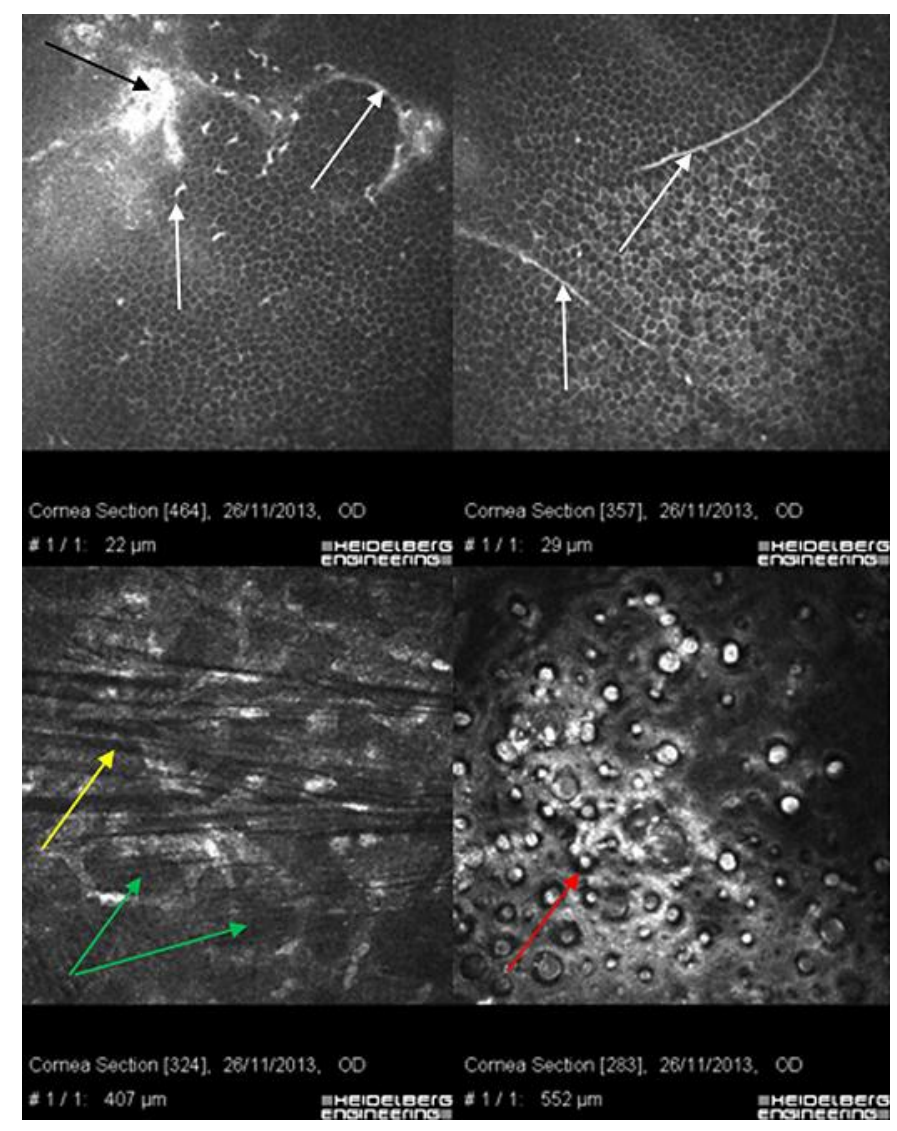

Fig. 3. Confocal microscopy of the right eye. Ring- and fingerprint-shaped EBMCD (white arrows); corneal deposit (black arrow); honeycomb corneal edema (green arrows); keratoconic dark microstriations (yellow arrow); FECD (red arrow). 
Case Reports in

Ophthalmology
Case Rep Ophthalmol 2014;5:281-288

DOI: $10.1159 / 000367937$

Mazzotta et al.: First Identification of a Triple Corneal Dystrophy Association: Keratoconus, Epithelial Basement Membrane Corneal Dystrophy and Fuchs' Endothelial Corneal Dystrophy

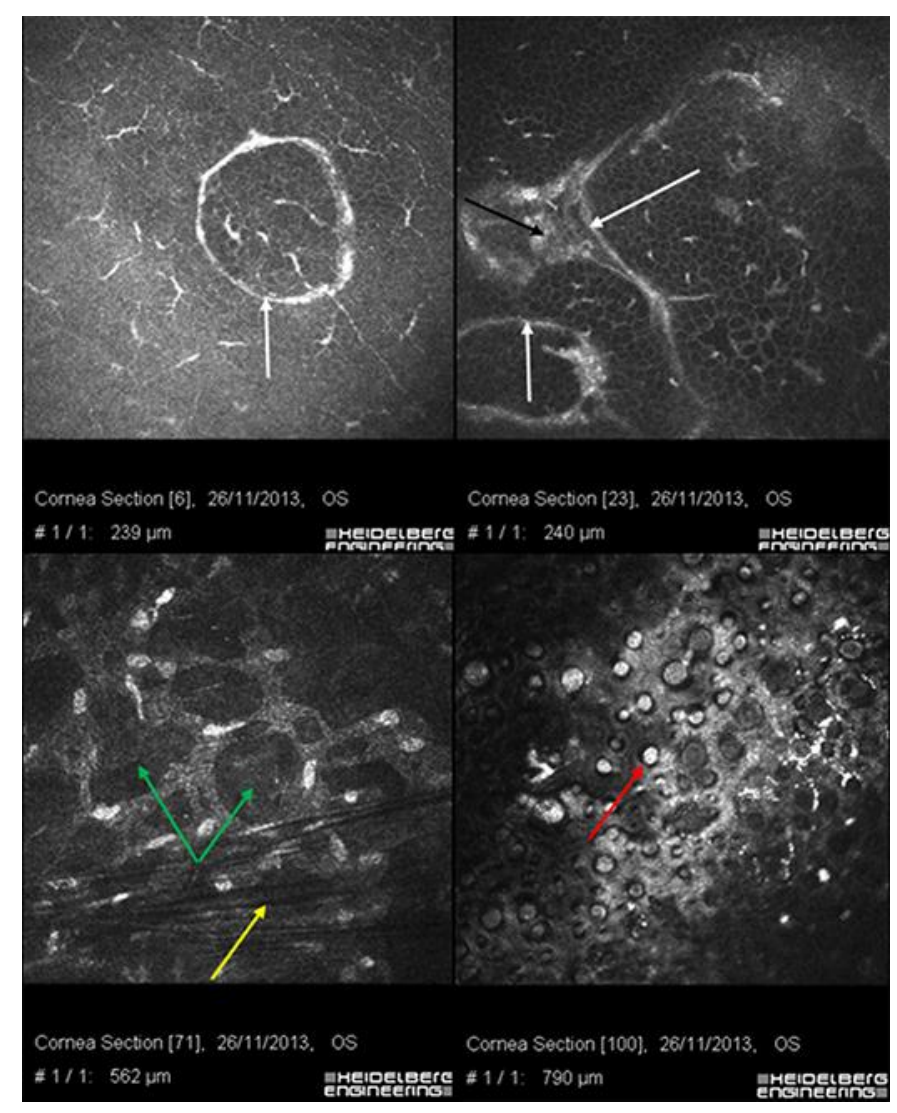

Fig. 4. Confocal microscopy of the left eye. Ring-shaped EBMCD (white arrows); deposits (black arrow); honeycomb corneal edema (green arrows); keratoconic dark microstriations (yellow arrow); FECD (red arrow). 